ANNA DAZBROWSKA

D HTTP://ORCID.ORG/ 0000-0002-8250-2762

Jagiellonen-Universität

e-mail: anna.barbara.dabrowska@uj.edu.pl

\title{
Poetik der Iranischen Revolution von 1978-1979 im Roman Die ersten Tage der Welt (2019) von Salem Khalfani
}

„Revolutions are not made. They come”

Wendell Philipps / Theda Skocpol ${ }^{1}$

\section{Abstract \\ Poetics of the Iranian Revolution of 1978-1979 in the novel Die ersten Tage der Welt (2019) by Salem Khalfani}

The aim of this paper is to examine how given literary devices correspond with the fatalistic portrayal of the Iranian Revolution of 1978-1979 in Salem Khalfani's novel Die ersten Tage der Welt. The fatalism, regarded as a social necessity and dependence of the drives, is emphasised by the theatrum mundi metaphor as well as the similes comparing the revolutionary events with the dynamic processes which can be found in the world of nature. The outcomes of the revolution are, however, connected with the poetics of lifelessness. Underneath the manifested layer of the novel, which emphasises the fatalism, there is also a covert layer of the text, which suggests that the narrator may be rationalising and denying any guilt for taking part in such a destructive mass event.

Keywords: Iranian Revolution, fatalism, poetics

Schlüsselwörter: Iranische Revolution, Fatalismus, Poetik

Der im Jahre 1963 in Iran geborene Autor Salem Khalfani kam 1985 in die Bundesrepublik Deutschland, wo er Literaturwissenschaft studier-

1 Th. Skocpol, Rentier State and Shi'a Islam in the Iranian Revolution, „Theory and Society" 1982, vol. 11, S. 265-283, hier S. 266. 
te. ${ }^{2} 2003$ veröffentlichte er eine Monografie unter dem Titel Ähnlichkeiten des Absurden, die Affinitäten zwischen dem Roman Das Schloss von Franz Kafka und dem Drama Warten auf Godot von Samuel Beckett nachweist. 2009 erschien seine Novelle Das valencianische Wasser und 2010 seine Gedichtsammlung Nachtschwimmer.

Im Vordergrund seines 2019 veröffentlichten Romans Die ersten Tage der Welt steht eine Liebesgeschichte: Der im iranischen Dorf Kaban lebende autodiegetische Erzähler namens Hamed ist von seiner Lehrerin fasziniert und betet sie selbst dann noch an, als ihr Arbeitsvertrag zu Ende geht und sie nach Teheran zurückkehrt. Schließlich bricht die Iranische Revolution von 1978-1979 aus und reißt die meisten Figuren mit sich. Nach der Revolution verlässt Hamed seine Heimat. Viel später besucht er wieder Kaban, was ihn zu Überlegungen über die Vergangenheit inspiriert.

Nach knappen Informationen zur Iranischen Revolution steht in der folgenden Analyse die Poetik im Vordergrund, die hier als eine bestimmte Art der Gestaltung des Narrativs der Revolution, ein Stil und eine spezifische Anwendung von Bildern verstanden wird. ${ }^{3}$ Zuerst werden Metaphern und Vergleiche untersucht, die eine fatalistische Darstellung der Revolution unterstützen. Mit Fatalismus ist hier die gesellschaftliche Notwendigkeit zu einem bestimmten Zeitpunkt und die Abhängigkeit von den dunklen menschlichen Trieben gemeint. Es soll nachgewiesen werden, dass die Poetik der revolutionären Ereignisse etwa dank dem Welttheatertopos und der Vergleiche mit Naturprozessen durchaus dynamisch ist. Dagegen ist die Poetik anders im Fall der Beschreibung der Revolutionsfolgen, die von Hamed veranschaulicht, aber überhaupt nicht kommentiert werden: Obwohl er keine Stellung zu politischen Konsequenzen der Revolution nimmt, weist die mit der Leblosigkeit verbundene Poetik, die in der Beschreibung der postrevolutionären Wirklichkeit vorherrscht, auf negative Entwicklungen in Iran hin.

Schließlich soll mithilfe ausgewählter Elemente der Tiefenhermeneutik aufgezeigt werden, dass es sich um einen Roman mit „doppeltem Boden ““4 handelt: Während der Erzähler mehrmals behauptet, dass er der Revolution blind folgen musste und es keine andere Möglichkeit gab (was als ein Legitimationsmuster bzw. eine Erklärung gilt), kann man auf der latenten Romanebene die Problematik der Schuld bzw. Verantwortung bemerken. Ausgehend von Überlegungen aus der Studie Die Welt als Bühne mit doppeltem Boden. Tiefenhermeneutische Rekonstruktion kultureller Inszenierungen Hans-Dieter Königs wird angenommen, dass Irritationen, also Inkonsistenzen im Text, auf eine verschleierte Sinnebene hinweisen können. Der latente Textsinn wird u.a. dank dem affektiven Verstehen

2 Mehr Informationen über den Autor sind u.a. in diesem Onlineartikel zu finden: V. Bolduan, Gespräch mit dem in Wiesbaden lebenden iranischen Autor Salem Khalfani, https://www.wiesbadener-kurier.de/freizeit/kunst-und-kultur/kulturnachrichten/gesprach-mit-dem-in-wiesbaden-lebendeniranischen-autor-salem-khalfani_20268584 (Zugriff: 12.01.2019).

$\mathrm{Zu}$ verschiedenen Bedeutungen des Begriffs Poetik vgl. D. Korwin-Piotrowska, Poetyka. Przewodnik po świecie tekstów, Kraków 2011, S. 16.

4 Vgl. die Studie: H.-D. König, Die Welt als Bühne mit doppeltem Boden. Tiefenhermeneutische Rekonstruktion kultureller Inszenierungen, Wiesbaden 2019. 
sichtbar. ${ }^{5}$ Er ist mit den Inhalten verbunden, die in der gegebenen Kultur (z.B. im postrevolutionären Iran, wo die Handlung des Romans von Khalfani spielt) nicht erwünscht sind.

\section{Die Iranische Revolution von 1978-1979}

Die Revolution von 1978-79 stürzte die iranische Monarchie. Zu ihren wichtigsten Ursachen zählten der autoritäre Regierungsstil des Schahs Mohammad Reza Pahlavi, die wirtschaftliche und politische Abhängigkeit Irans von Fremdmächten wie den USA., die weitgehende Übernahme der westlichen kulturellen Muster, eine zu schnelle Modernisierung des Landes und die Kluft zwischen Reich und Arm. Sieger der Revolution war Ajatollah Ruhollah Chomeini, der mit seinen schiitischen Anhängern die Islamische Republik Iran gründete und somit die Theokratie einführte. Die Revolution bedeutete eine wichtige Zäsur für Iran und die Region, denn sie trug zur radikalen Abkehr vom politischen Kurs der Monarchie und somit von der westlichen Welt bei. ${ }^{6}$

\section{Fatalismus und dynamische Poetik der revolutionären Ereignisse}

Laut Hamed, der zurzeit des Revolutionsausbruchs in der siebten Klasse ist, bildet die Tätigkeit einer „mächtigen Hand“ den einzigen Revolutionsgrund, wozu er sich später retrospektiv folgendermaßen äußert:

Kriege werden von Völkern ausgeführt, aber nie von ihnen geführt. Und Völker sind nie imstande, einen Krieg anzuzetteln oder den bereits begonnenen zu beenden. Erst eine Hand aus einem Versteck, eine mächtige aber verborgene Hand, kann einen Krieg inszenieren oder ihm ein Ende geben. Doch dann müssen die Hände der Durchschnittsmenschen mitmachen, sonst kommt es zu nichts. Und wenn die verborgene mächtige Hand da ist, dann machen ja alle mit. Und der Krieg nimmt seinen Lauf; er kann erst dann zu Ende gehen, wenn diese jenseitige starke Hand den Stoppbefehl gibt. Völker können keinen Krieg anzetteln und keinen zu Ende bringen. Aber sie können und sie müssen ihn ausführen. [...] Und so ist es auch mit Revolutionen. ${ }^{7}$

In der angeführten Passage erscheinen Völker als keine entscheidungsfähigen Subjekte. Die Notwendigkeit ihrer Taten wird durch das Modalverb „müssen" ausgedrückt. Aus dem Zitat geht hervor, dass die mächtige Hand einen

5 Vgl. ibid., S. 34. Im vorliegenden Artikel werden nur die im Haupttext genannten Punkte und nicht das ganze methodologische Schema aus der Studie Königs übernommen, welches zu einem beträchtlichen Teil auf A. Lorenzer rekurriert. (Lorenzer hat u.a. das Modell des szenischen Verstehens entwickelt, welches mit der tiefenhermeneutischen Kulturanalyse verbunden ist). Es werden hier nur solche Aspekte gewählt, die wichtig für die Untersuchung des Themas der vorliegenden Arbeit sind.

$6 \mathrm{Zu}$ den Forschern, die sich mit der Iranischen Revolution befassen, gehören u.a. E. Abrahamian und H. Dabashi. Vgl. z.B. ihre Studien: E. Abrahamian, A History of Modern Iran, Cambridge 2008; H. Dabashi, Theology of Discontent: The Ideological Foundation of the Islamic Revolution in Iran, New York 2017.

7 S. Khalfani, Die ersten Tage der Welt. Roman, Bremen 2019, S. 145f. Im weiteren Verlauf wird auf diese Ausgabe mithilfe der gegebenen Seitennummer in Klammern hingewiesen. 
Krieg - und man kann hinzufügen: auch eine Revolution - inszeniert, was Assoziationen zu einem Theaterstück oder Film auslöst. ${ }^{8}$ Man kann dabei an den Topos des Welttheaters denken, den u.a. Platon verwendete. Er stellte das Leben als Schauspiel und Menschen als Marionetten in göttlichen Händen dar (vgl. den Begriff theatrum mundi). ${ }^{9}$ Der Topos ist auch in der persischen Kultur auffindbar, worauf z.B. die folgenden Verse eines Gedichts ${ }^{10}$ von Omar Chajjam (ca. 1048 - ca. 1131) hinweisen: „Wir sind die Marionetten und das Firmament der Puppenspieler, / das ist kein Gleichnis, sondern Wirklichkeit; / eine Zeit lang spielten wir in dieser Vorstellung, / ehe wir in der Truhe der Vergessenheit verschwinden". ${ }^{11}$

Der Topos des Welttheaters kann nicht nur einer anschaulichen Darstellung einer Revolution, sondern auch einer Weltdeutung dienen, die je nach Kontext und ideologischer Haltung des jeweiligen Autors unterschiedlich ausfallen kann. Die Welttheaterkonzepte divergieren z.B. hinsichtlich der Frage, wer Regisseur des Schauspiels ist und wer die Schauspieler sind. ${ }^{12}$

Es ist überlegenswert, wer oder was Regisseur im Roman Khalfanis ist. Die Phrase ,jenseitige Hand“ löst meistens Assoziationen zur Transzendenz aus. Es kann sich hier aber um ,jenseits“ im Sinne einer räumlichen Bestimmung handeln, also darum, was sich außerhalb des Dorfes befindet. Es wird zum Empfänger der großen sozialen Bewegungen, die ihren Ursprung woanders haben. Man erfährt nicht, welche mächtige Instanz die Entscheidung über die Revolution an einem anderen Ort fällt. Auf jeden Fall geht aus dem unten angeführten Zitat hervor, dass die revolutionären Ideen aus einem Zentrum, also aus Teheran, kommen und in die Provinz vordringen. Ihr Ursprung liegt aber noch weiter weg, sodass die Vorstellung von der Hauptstadt als Zentrum überschritten wird. Es handelt sich um den Beginn einer neuen Ära (vgl. S. 149) ${ }^{13}$ und die Verbreitung globaler Tendenzen $^{14}$, zu denen z.B. die allmähliche Abschaffung der Monarchie im 20. Jahr-

8 Die mit dem Theater verbundene Metaphorik ist auch z.B. in der Phrase ,das Spektakel der Sonnenaufgänge" (S. 32) sichtbar.

9 Vgl. dazu Chr. Leiteritz, Revolution als Schauspiel. Beiträge zur Geschichte einer Metapher innerhalb der europäisch-amerikanischen Literatur des 19. und 20. Jahrhunderts. Komparatistische Studien, Beihefte zu ,arcadia”. Zeitschrift für Vergleichende Literaturwissenschaft, hg. von M. Moog-Grünewald und J. Wertheimer, Bd. 18, Berlin-New York 1994, S. 11, 279.

10 Es handelt sich um Rubai (Plural Rubaiyat), also eine Gedichtform mit vier Zeilen. Der erste, zweite und vierte Vers reimen sich. Es können sich aber auch alle Verse reimen.

11 O. Chajjam, Wie Wasser strömen wir, in: Hafis, Rumi, Omar Chajjam. Die schönsten Gedichte aus dem klassischen Persien, Hg. K. Scharf, übertragen von C. Atabay, München 2004, S. 7-33, hier S. 18. Zur Bedeutung des Puppentheaters in der persischen Kultur vgl. den Band: The Marionettes by Bahrām Beyza'i, Salzburg 2005, Hg. J.-A. George, P. Loloi, G. Pursglove. Ich danke Amir Goodarzi für den Hinweis auf Chajjam und Beyza'i.

12 Vgl. dazu Chr. Leiteritz, op. cit., S. 6-22.

13 Wenn man an die außerliterarische Wirklichkeit denkt, bemerkt man die Ironie des Schicksals: Die Revolution, die eine neue Ära einleiten sollte, mündete in einer konservativen, zum großen Teil vergangenheitsorientierten Wende in Iran.

14 Ich bin Salem Khalfani für den folgenden Mail-Kommentar zu der irdischen und nicht theologischen Bedeutung der mächtigen Hand und des Begriffs ,,jenseits“ dankbar: „Vielleicht spricht er [d.h. Hamed] von dem neuen globalen System, das über die Städte ins Dorf eindringt und daher mit 
hundert in verschiedenen Weltteilen gehörte. In der folgenden Aussage Hameds sind es nicht die Menschen, die die Revolution anstreben, sondern die Revolution kommt vielmehr zu ihnen:

Der Geruch der Revolution kam zu uns über Radios und Fernseher, die ihren Weg allmählich ins Dorf fanden [...]. Das Neue, noch nicht Gesehene, kam über die Straße, die nach Norden, zweihundert Kilometer weiter in die Stadt Bushehr führte, und - das wussten wir allmählich - von dort in eine andere Stadt und so weiter bis nach Teheran, und von dort bis überallhin. Dieser Geruch war so intensiv, so penetrant, dass jeder ihn roch. Alte und Junge, Kranke und Gesunde, Blinde und Sehende. Man musste nur riechen können (S. 148f.).

Laut Duden tritt das Verb ,riechen“ z.B. in der folgenden Wendung auf: „〈in übertragener Bedeutung:) er roch (umgangssprachlich; merkte) sofort, dass hier etwas nicht stimmte”. ${ }^{15}$ Mithilfe dieses Verbs und des Substantivs „Geruch“ kann man also auf intuitive Wahrnehmung und Spürsinn hinweisen. Geruch ist etwas, das man einatmet, das also von außen kommt und ins Innere dringt. ${ }^{16}$ Nach der angeführten Passage musste man also nur sensibel für politische bzw. soziale Bewegungen sein, um sich der Revolution anzuschließen. Aus den Aussagen Hameds geht hervor, dass fast alle es waren und sich ohne jegliche Überlegungen an der Revolution beteiligten. Dabei gelten Radio und Fernsehen wenigstens teilweise als Regisseure des Welttheaters.

Hamed betont in der folgenden Beschreibung der Massenereignisse, dass die Taten der Figuren von außen beeinflusst werden:

Und sobald diese Hand die Fahne mit dem markanten Wort REVOLUTION erhebt, kann jeder dieses Wort lesen, dann gibt es nur wenige, die sich der Tragweite der mächtigen Hand entziehen können. Deshalb denke ich, dass kein Volk ein Held ist, weder in den Kriegen noch in den Revolutionen noch in Friedenszeiten. Im Guten wie im Schlechten. Ein Volk ist immer das Opfer (S. 146).

Demnach wird das Heroische aus der Revolutionsgeschichte eliminiert, aber zugleich - wenn man weiter denkt - die Täterschaft der aufgewühlten Masse. Die Schuld des Volkes wird ganz ausgeklammert und seine Vertreter erscheinen lediglich als Leidtragende.

Die Revolution gewinnt „himmlisches Ausmaß” (S. 146), was Assoziationen mit dem Glauben an den Zusammenhang von Schicksal und Himmelskörpern in der persischen und europäischen Tradition aufkommen lässt. Etwa im persischen Epos Schahnameh von Firdausi (ca. 940-ca. 1020) korrespondiert die Wende im Schicksal der Helden mit astronomischen Prozessen. ${ }^{17}$ Auch in der europäischen Kultur sah man u.a. im 16. Jh. oft einen Zusammenhang der Astronomie mit poli-

dem Jenseitigen auf das hinweist, was nicht zum Dorf gehört. Die Hand ist auch mit dem Dunklen, Triebhaften verbunden".

15 (dostęp: 27. Juli 2020) https://www.duden.de/rechtschreibung/riechen.

16 Hamed stellt im nächsten Abschnitt fest, dass der Revolutionsgeruch doch von innen kam. Möglicherweise meint er damit, dass die Revolution niedrige Instinkte in den Menschen enthemmte.

17 Vgl. H. Ringgren, Fatalism in Persian Epics, Uppsala Universitets Årsskrift 1952, 13, „Acta Universitatis Upsaliensis", Wiesbaden 1952, S. 52, 65, 73-78. 
tischen Entwicklungen. ${ }^{18}$ Der Glaube an Astrologie ist heute wenig verbreitet und der Roman versucht ihn anscheinend nicht zu beleben. Die Hyperbel ,himmlisches Ausmaß" mag jedoch durch Assoziationen zur Astrologie den Eindruck des Fatalismus steigern.

Das Notwendige und Unabänderliche der Ereignisse sowie die Unschuld der gelenkten Masse wird auch mithilfe der Vergleiche aus der Naturwelt hervorgehoben $^{19}$, die die Poetik der revolutionären Ereignisse gestalten. Sie drücken das hohe Tempo der Geschehnisse aus und heben die Beweglichkeit der umtriebigen Figuren hervor. Der Verlust ihrer bisherigen Identität wird mithilfe eines Vergleichs mit dem Sturm - der u.a. aus den Darstellungen der Französischen Revolution bekannt ist $^{20}$ - in der folgenden Aussage Hameds veranschaulicht:

Ich wusste, dass die Revolution wie ein Sturm ist, der die Vögel von einem Baum zu einem anderen, zuweilen zu einem ganz entfernten Baum treiben, sie heimatlos machen kann, falls sie ihn überlebten. Oder wie ein Erdbeben, das sie alle auf einmal aus den Ästen in die Luft vertreibt (S. 149).

Abgesehen von dem Vergleich mit einem Erdbeben, welches in den Darstellungen von Revolutionen eher auf Katastrophe als auf Regeneration bzw. Erlösung hinweist $^{21}$, betont die Metapher der Wellen die Dynamik der gesellschaftlichen Prozesse, was in den folgenden Aussagen Hameds sichtbar ist:

Und bevor wir Gelegenheit bekamen, festzustellen, ob die Gerüchte nur Gerede waren oder ob dahinter wirklich etwas Handfestes steckte, befanden wir uns in ihrer Mitte und waren selbst Teil von ihnen. Wir wurden vom Sog ihrer Wellen mitgerissen. Wir waren selbst ein Gerücht (S. 150).

in dieser Welle waren wir alle eins geworden, eine schäumende Welle, die der Vergangenheit Adieu sagte und sich gewaltig auf eine ganz neue und ungewisse Zukunft zubewegte (S. 153).

In den beiden Zitaten wird der Verlust der Individualität thematisiert. In einer Welle bildet das Wasser eine Einheit und man sieht keine Tropfen, sodass sich die Metapher gut zur Veranschaulichung der kollektiven Handlungen eignet. Während Hamed früher als ein Außenseiter das Personalpronomen ,ich“ verwendete, benutzt er jetzt vor allem die Plural-Form ,wir“.

Die Figuren folgen der Welle von Gerüchten, was darauf hinweisen mag, dass die Revolution sich u.a. über den Weg der Mundpropaganda verbreitet. Zuerst ist die revolutionäre Bewegung noch weit entfernt und man bekommt wahrscheinlich nur vage Informationen darüber. Dann erfasst sie aber auch die Pro-

18 Vgl. dazu C. Brosseder, Im Bann der Sterne: Caspar Peucer, Philipp Melanchthon und andere Wittenberger Astrologen, Berlin 2004, vor allem das Kapitel Politische Wirklichkeit und politischer Anspruch der Astrologie, S. 27-80. (Am Rande kann man anmerken, dass der Begriff Revolution bei Kopernikus mit sich wiederholenden Kreisbewegungen von Himmelskörpern verbunden war).

19 Chr. Leiteritz stellt dies bei der Untersuchung ausgewählter Darstellungen der Revolution in der Literatur fest. Vgl. dazu Chr. Leiteritz, op. cit., S. 24.

20 Vgl. dazu ibid., S. 23f.

21 Vgl. P. Sztompka, Agency and Revolution „International Sociologyee 1990, vol. 5, no. 2, S. 129-144, hier S. 131. 
vinz. Ihre Bewohner werden selbst zu Protagonisten von Gerüchten, und zwar vermutlich in anderen Orten, in die die Revolution im Rahmen ihres Wachstums erst gelangen wird. ${ }^{22}$ Außerdem kann das Wort „Gerücht“ die Desorientierung der Figuren betonen: Sie wissen nicht, was stimmt, und sie können ihre Lage nicht richtig einschätzen.

Als die Revolution Kaban ergreift, verlieren eigene Wünsche und Vorstellungen an Bedeutung (vgl. S. 150). Hamed marschiert mit anderen Schülern und schreit Parolen gegen den Schah, weil andere es so machen und auch in ihm dunkle Triebe aktiv werden. Er beschreibt den allgemeinen Destruktionsdrang folgendermaßen:

In den Städten wurden Banken, Gendarmerien und Schlösser angegriffen. Doch in unserem Dorf gab es nichts Staatliches außer der Schule. Wir konnten also nichts angreifen, es gab nichts, das wir zerstören konnten, und das war ärgerlich. Wir demonstrierten nur, wir versuchten lauter und lauter zu schreien. Wir besorgten uns Stangen und rissen alle Shah-Bilder aus unseren Büchern und befestigten sie daran (S. 152f.).

Während die von der Zerstörungswut besessenen Revolutionäre in Städten große Schäden anrichten, können es die Provinzbewohner mit ihnen - in dieser Hinsicht - nicht aufnehmen. Sie sind frustriert über ihre „Unfähigkeit, irgendetwas zu zerstören" (S. 154). Die einzige Möglichkeit der Destruktion von öffentlichen Symbolen bieten Schulbücher und die Schule. Ihre Zerstörung wird in einem kurzen Dialog kritisch hinterfragt. Es fallen hier keine Namen, sodass man nicht weiß, welche Figur sich jeweils äußert. Jemand fragt die Schüler, wieso sie ihre Schule demolierten und ob sie sich dessen bewusst seien, dass sie im Winter frieren müssen.

Die Schüler wissen nicht einmal, wer den ersten Stein auf eine Fensterscheibe geworfen hat. In der Masse ist dies unwichtig, weil alle gemeinsam agieren. Wenn die Steine das Glas zerbrechen, verschwinden auch die Spiegelbilder der Schüler darauf. Ihre Widerspiegelung hat etwas Unheimliches an sich, was in der folgenden Aussage Hameds zum Ausdruck kommt:

Es war so merkwürdig, denn mir schien, dass die Gestalten, die fielen, den Krach verursachten. Und es war so, als ob nicht wir die Scheiben mit Steinen bewarfen und sie zerschlugen, sondern es waren die geisterhaften Gestalten, die am hellichten Tag in den Fenstern hinter den Scheiben im Dunkeln der Klassen lebten und sich so energisch hin und her bewegten. Bei jedem Stein, den ich warf und mit dem ich ein Fenster zerschlug, hatte ich das Gefühl, dass es nicht meine Hand war, die dies beging (S. 155).

Man hat den Eindruck, dass es sich um gespenstische Doppelgänger handelt. Hamed fühlt sich uneins mit sich selbst, er identifiziert sich nicht mit seinen eigenen destruktiven Taten. Affekte gewinnen Oberhand.

22 Laut der Studie Masse und Macht von Elias Canetti ist für die Masse u.a. typisch, dass sie immer wachsen will. Vgl. E. Canetti, Masse und Macht, Hamburg 1960, S. 30. Dies ist auch im Roman Khalfanis sichtbar. 


\section{Revolutionsfolgen und die Poetik der Leblosigkeit}

Während die Steine bei dem Angriff auf die Schule in Bewegung sind, verkörpern sie ansonsten in der Regel das Unveränderbare. Hamed bemerkt einen Kontrast zwischen ihrer Beständigkeit ${ }^{23}$ und der Vergänglichkeit des menschlichen Lebens. Er schätzt die Steine als Zeugen der Geschichte, die die Vorbeigehenden an die Vergangenheit erinnern, aber er bemerkt zugleich ihren Zusammenhang mit dem Friedhof. Dies ist in seiner folgenden Aussage sichtbar: „Jeder Mensch besitzt einen oder mehrere Steine, auch nach dem Tod. Die Verwandten eines Verstorbenen wissen es ganz genau, sie stellen also diese Steine auf sein Grab“ (S. 158). Wenn Hamed viele Jahre nach der Revolution nach Kaban reist, besucht er die Begräbnisstätte seiner Schulkameraden. Sie verloren ihr Leben in der Revolution oder im Ersten Golfkrieg (1980-1988), der als eine der Konsequenzen der Revolution gelten kann. ${ }^{24}$ Am Grab Alis befindet sich die Inschrift „Märtyrer”, was auf seinen Tod im Krieg gegen Irak hinweist. Die Symbolik von Grab und Friedhof betont eine enge Verbindung der Revolution und des Kriegs mit dem Todestrieb und sie beeinflusst affektiv die Leser.

Außerdem besucht Hamed die Schule, die seit Jahren nicht mehr benutzt wird und bald abgerissen werden soll. Er geht durch das verrostete und bröselnde Eisentor. In einem Klassenraum sieht er eine verblasste Tafel sowie ein verstaubtes Fensterglas und er atmet die Luft der Vergangenheit ein. Während die Schule früher mit Leben erfüllt war, wirkt sie jetzt wegen ihrer Leere unheimlich.

Schließlich verkörpert das zerstörte Haus der Lehrerin die verhängnisvollen Folgen der Revolution und des Kriegs. Die Ruine, die Gräber der Schulkameraden und die verlassene Schule gehören zu den Orten, die eine bedrückende Stimmung evozieren und die Abwesenheit von Klassenkameraden und der Lehrerin spüren lassen - auch wenn Hamed das Bewusstsein ihrer Absenz abwehren will, indem er ihre Präsenz imaginiert. In den Beschreibungen der genannten Orte dominiert das Statische und die Leere.

\section{Manifeste und latente Romanebene}

Hamed fühlt sich für nichts verantwortlich. Als er seinen Mitschüler Ali angreift und seine Augen dauerhaft beschädigt, behauptet er, dass eine fremde, mächtige Hand ihn dazu zwang. Jahrelang später spricht er über seine Auswanderung, als ob er keinen Einfluss darauf gehabt hätte. Dies ist in seinem folgenden Gespräch mit Hamid, einer Nebenfigur, erfahrbar: „Er fragte mich, ob es im Ausland besser wäre als hier. Ich erklärte ihm, dass das nicht meine Entscheidung war" (S. 64).

23 Auch Mundi, ein Klassenkamerad Hameds mit Behinderung, verkörpert sie wegen seiner Bewegungseinschränkungen.

${ }^{24}$ Irak hatte Angst vor der Verbreitung der Revolution außerhalb der iranischen Grenzen. Außerdem war Iran nach der Revolution geschwächt, was ihn zu einem leichten Aggressionsobjekt machte. Es gab aber auch andere, historische Ursachen der Eskalation, wie z.B. territoriale Konflikte. 
Der Leser bzw. die Leserin erfährt nicht, wieso Hamed seine Heimat verlassen hat. Er sagt nur noch, dass er vielleicht nach Kaban zurückziehen wird.

Nur einmal stellt er sich die Frage nach der Schuld im Kontext der Revolution, die er aber bald für sinnlos hält:

Ich fragte mich, ob wir mit ruhigem Gewissen leben können, wenn uns bewusst ist, dass wir diese jungen Leute aus einem anderen Zustand in diesen neuen gebracht und ihnen eine neue Ära verschafft haben. Eine Ära, in der sie in eigenen Heimatstädten und -dörfern fremd waren, ohne dass sie es wussten, ohne, dass sie sich beklagten. Doch wenn ich an jene Hand denke, die stärker war als wir und als unser Wille, dann bin ich mir sicher, dass diese Fragen [d.h. die Fragen nach der Schuld] absolut überflüssig sind (S. 157).

Hamed rationalisiert sein Handeln und verleugnet die Schuld der sich an der Revolution beteiligenden Kabaner. ${ }^{25}$ Interessanterweise erwähnt er gar nicht sein geringes Alter zurzeit der Revolution, welches die Schuldfrage in der Tat relativieren könnte, sondern er verweist nur auf die Befehle der mächtigen Hand. Zusammenfassend lässt sich feststellen, dass er die Unschuld mehrmals verbalisiert.

Im angeführten Zitat erwähnt er die Entfremdung der Kabaner von ihrer bisherigen Umgebung, aber er kritisiert ansonsten gar nicht das postrevolutionäre politische System in Iran. Obwohl jeglicher Kommentar dazu fehlt, gibt es einige Hinweise auf verhängnisvolle Folgen der Revolution: Hamed verfolgt regelmäßig Zeitungsbeiträge über Selbstmörderinnen in Iran, um zu erfahren, ob seine Lehrerin sich für den Freitod entschieden hat. ${ }^{26} \mathrm{Im}$ Roman steht nichts darüber, dass Frauenrechte nach der Revolution weitgehend eingeschränkt wurden. Hamed erwähnt nur, dass viele Frauen an einer Überdosis von Medikamenten sterben oder sich aus einem Fenster stürzen. Eine gewisse Irritation entspringt dem Kontrast zwischen dem Verschweigen der politischen und sozialen Lage der iranischen Frauen und den Überlegungen, ob die Lehrerin sich vielleicht getötet hat:

Aber warum sollte sie [d.h. die Lehrerin] Hand an sich legen? Warum sollte sie so sterben und nicht anders? Das war nur ein Gefühl ohne jegliche Logik (aber welches Gefühl hat schon Logik?). Und es kam trotzdem nicht selten vor, dass eine Person wie sie nur in dieser Art und Weise aus der Welt schied (S. 163f.)

Hamed lässt politische Folgen der Revolution aus, aber seine Vorstellungen werden anscheinend davon doch beeinflusst, was mit der Poetik der Indirektheit verbunden ist. Er verdrängt das Problem der Verantwortung für das postrevolutionäre System, welches jedoch in seinem Unbewussten zum Ausdruck kommt. Während er als Junge kaum etwas von der Politik wissen konnte, ist er nach dem Ersten Golfkrieg ein Erwachsener, der sich zur Politik überraschenderweise gar nicht äußert. Die Ahnung, dass seine geliebte Lehrerin vielleicht Selbst-

25 In der Erzählung Hameds spielt das Unheimliche eine bedeutende Rolle, aber es schließt die Möglichkeit einer kohärenten und plausiblen Erklärung der Revolution nicht aus.

26 Hamed überlegt, ob er an ihren Selbstmord denkt, um sie in Gedanken für sich alleine zu retten (Vgl. S. 163). Er stellt fest: „Das mag sein“(S. 163). Diese Erklärung muss ihm jedoch unbefriedigend vorkommen, denn er fragt erneut, wieso er sich mit ihrem hypothetischen Freitod befasst (vgl. das angeführte Zitat im Haupttext). 
mord begangen hat, kann jedoch auf eine Beschäftigung mit dem postrevolutionären politischen System auf der latenten Romanebene hinweisen.

Ebenfalls die Worte eines unbekannten Blinden, der Hamed das zerstörte Haus der Lehrerin zeigt, könnten auf die Schuldfrage hinweisen: „[D]as ist das, was Sie gesucht haben" (S. 172). Diese Aussage mag sich auf die Adresse beziehen, aber sie kann auch im übertragenen Sinne gedeutet werden: Die Revolutionäre haben schließlich nur die Destruktion angestrebt. Suchen ist eine aktive Tätigkeit, sodass die Passivität der Revolutionäre an dieser Stelle infrage gestellt wird. Dank solcher Aussagen können die Behauptungen Hameds von der Unschuld der Revolutionäre hinterfragt werden.

Schließlich stellt Hamed während seines Besuchs in Kaban fest, dass man sich dort mit niemandem über die Vergangenheit unterhalten kann. Dies entspringt dem Tod und Verschwinden vieler Kabaner. Man erfährt nicht, ob manche von ihnen aus politischen Gründen abwesend sind, sodass die Romanstelle rätselhaft bleibt. Des Weiteren drängt sich bei der Lektüre der Worte Hameds die Frage auf, ob das Vergessen nur die Folge der zeitlichen Distanz ist oder ob man es eher mit dem allgemeinen Verschweigen von bestimmten Themen in Iran zu tun hat:

Ali und viele andere lebten nicht mehr. Und viele sind irgendwie verschwunden, spurlos und für immer weg. Die Leute, die unser altes Haus gekauft hatten und seit Jahrzehnten darin einquartiert waren, können sich kaum an etwas erinnern. Und die anderen, die Nachkommen von den damaligen Nachbarn, genauso wenig (S. 69f.).

Die latente Dimension des Romans und die Poetik der Indirektheit scheinen in diesem Fall mit der in der Islamischen Republik Iran unerwünschten Kritik der postrevolutionären Wirklichkeit verbunden zu sein. ${ }^{27}$ Die allgemeine Amnesie der Bewohner Kabans bewirkt eine Irritation, denn sie wirkt kaum wahrscheinlich. Die zitierte Passage mag auf die Verdrängung der Schuld durch die Kabaner hinweisen, die sich an die Revolutionszeit nicht mehr erinnern wollen und das Wissen darüber an ihre Nachkommen nicht weitergegeben haben.

\section{Schlussfolgerungen}

Auf der manifesten Ebene präsentiert der Roman kaum Reflexionen über politische Ursachen und Folgen der Revolution. Obwohl Hamed das zerstörte Haus, den Tod seiner Klassenkameraden und die hohe Suizidrate unter Frauen gar nicht kommentiert, weisen sie auf emotional belastende Folgen der Revolution hin. Anscheinend rationalisiert Hamed die Teilnahme der Kabaner an der Revolution, um sich nicht verantwortlich für die schlimme politische Entwicklung in Iran fühlen zu müssen. Er verdrängt die Frage nach dem menschlichen Beitrag zu einschneidenden Prozessen mithilfe der fatalistischen und zugleich dynamischen

27 Der Roman ist auch mit der deutschen Kultur verbunden, aber die Untersuchung dieses Aspekts würde den Rahmen der vorliegenden Arbeit sprengen. Er erschien in Deutschland und ist u.a. für LeserInnen aus deutschsprachigen Ländern bestimmt, jedoch liegt sein Fokus auf dem Iran nach der Revolution. 
Bilder, die zur manifesten Textebene gehören und mit der gesellschaftlichen Notwendigkeit zu einem bestimmten Zeitpunkt sowie mit menschlichen Trieben verbunden sind. Weil die Figuren aus der Provinz ihm zufolge so handeln mussten, wie die mächtige Hand es von ihnen verlangte, sind sie ganz unschuldig als Marionetten auf der Weltbühne. Die Notwendigkeit ihrer Taten wird auch mithilfe des metaphorischen Stils ausgedrückt, der u.a. auf die Naturwelt rekurriert.

Nach der Revolution kann sich jedoch Hamed mit den Folgen des aus seiner Perspektive Unvermeidbaren nicht identifizieren. Er bemerkt, dass das Zusammengehörigkeitsgefühl der Kabaner nur eine kurzlebige Illusion war. Seine Gedanken kreisen um die Vergangenheit, deren Spuren mit der statischen, reglosen Poetik verbunden sind.

Trotz des mehrmals verbalisierten Glaubens an Fatalismus im oben genannten Sinn hat man den Eindruck, dass der Roman die Leser und Leserinnen durch seine latente Ebene doch zum kritischen Nachdenken über die Rolle der sich an Massenphänomenen beteiligenden Menschen, und zwar vor allem über die Verantwortung bzw. Schuld der Mitläufer und die dunkle menschliche Natur inspirieren möchte.

\section{Literaturverzeichnis}

\section{Primärliteratur}

Khalfani S., Die ersten Tage der Welt. Roman, Bremen 2019.

\section{Sekundärliteratur}

Abrahamian E., A History of Modern Iran, Cambridge 2008.

Bolduan V., Gespräch mit dem in Wiesbaden lebenden iranischen Autor Salem Khalfani, https:/www.wiesbadener-kurier.de/freizeit/kunst-und-kultur/kulturnachrichten/gesprach-mit-dem-in-wiesbaden-lebenden-iranischen-autor-salem-khalfani_20268584 (12.01.2010).

Brosseder C., Im Bann der Sterne: Caspar Peucer, Philipp Melanchthon und andere Wittenberger Astrologen, Berlin 2004.

Canetti E., Masse und Macht, Hamburg 1960.

Chajjam O., Wie Wasser strömen wir [in:] Hafis, Rumi, Omar Chajjam. Die schönsten Gedichte aus dem klassischen Persien, Hg. K. Scharf, übertragen von C. Atabay, München 2004.

Dabashi H., Theology of Discontent: The Ideological Foundation of the Islamic Revolution in Iran, London-New York 2017.

König, H.-D., Die Welt als Bühne mit doppeltem Boden. Tiefenhermeneutische Rekonstruktion kultureller Inszenierungen, Reihe Kritische Sozialpsychologie, hg. u.a. von M. Brunner; N. Burgermeister, Wiesbaden 2019.

Korwin-Piotrowska D., Poetyka. Przewodnik po świecie tekstów, Kraków 2011.

Leiteritz Chr., Revolution als Schauspiel. Beiträge zur Geschichte einer Metapher innerhalb der europäisch-amerikanischen Literatur des 19. und 20. Jahrhunderts. 
Komparatistische Studien, Beihefte zu ,arcadia”. Zeitschrift für Vergleichende Literaturwissenschaft, Hg. M. Moog-Grünewald; J. Wertheimer, Bd. 18, Berlin, New York 1994.

Ringgren H., Fatalism in Persian Epics, Uppsala Universitets Årsskrift 1952, 13, „Acta Universitatis Upsaliensis”, Wiesbaden 1952.

Skocpol Th., Rentier State and Shi'a Islam, ,Theory and Society ${ }^{\mathrm{ee}}$ 1982, vol. 11.

Sztompka P., Agency and Revolution, „International Sociologyee 1990, vol. 5, no. 2. The Marionettes by Bahrām Beyza'i, Hg. J.-A. George, P. Loloi, G. Pursglove, Salzburg 2005. 